\title{
Small and medium enterprise business solutions using data visualization
}

\author{
Norhaslinda Kamaruddin ${ }^{1}$, Raja Durratun Safiyah ${ }^{2}$, Abdul Wahab ${ }^{3}$ \\ ${ }^{1,2}$ Advanced Analytics and Engineering Centre, Faculty of Computer and Mathematical Sciences, \\ Universiti Teknologi MARA, Malaysia \\ ${ }^{1}$ Faculty of Science and Technology, C Campus, Universitas Airlangga, Indonesia \\ ${ }^{3}$ Kulliyah of Information and Communication Technology, International Islamic University Malaysia, Malaysia
}

\begin{tabular}{l}
\hline \hline Article Info \\
\hline Article history: \\
Received Jan 3, 2020 \\
Revised Mar 14, 2020 \\
Accepted Apr 2, 2020 \\
\hline
\end{tabular}

Keywords:

Business intelligence

Data analytics map

Data visualization

Dashboard

Small-medium enterprise

\begin{abstract}
The small and medium enterprise (SME) companies optimize performance using different automated systems to highlight the operations concerns. However, lack of efficient visualization in reporting results in slow feedbacks, difficulties in extracting root cause, and minimal corrective actions. To complicate matters, the data heterogeneity has intensely increased, and it is produced in a fast manner making it unmanageable if the traditional methods of analytics are applied. Hence, we propose the use of a dashboard that can summarize the operational events using real-time data based on the data visualization approach. This proposed solution summarizes the raw data, which allows the user to make informed decisions that can give a positive impact on business performance. An interactive intelligent dashboard for SME (iid-SME) is developed to tackle issues such as measurement of cases completed, the duration of time needed to solve a case, the individual performance of handling cases and other tasks as a proof of concept. From the result, the implementation of the iid-SME approach simplifies the conveyance of the message and helps the SME personnel to make decisions. With the positive feedback obtained, it is envisaged that such a solution can be further employed for SME improvement for better profit and decision making.
\end{abstract}

This is an open access article under the CC BY-SA license.

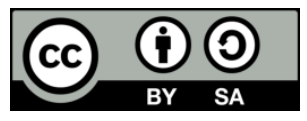

\section{Corresponding Author:}

Norhaslinda Kamaruddin,

Advanced Analytics and Engineering Center,

Faculty of Computer and Mathematical Sciences,

Universiti Teknologi MARA,

40450 Shah Alam, Selangor, Malaysia.

Email: norhaslinda@fskm.uitm.edu.my

\section{INTRODUCTION}

Small and medium enterprise (SME) in Malaysia is recognized as a supporting source of development and accepted as an established substructure for the fast-economic growth of the country [1]. Microenterprise across all sectors can be defined as firms with a sales turnover of less than RM 300,000 or employs less than 5 full-time employees [2]. For services sectors, a small enterprise is defined for firms with sales turnover ranging between RM 300,000 to RM 3 million or employs full-time employees between 5 to 30 staff. For a medium enterprise, the cut off for sales turnover is between RM 3 million to RM 20 million or having full-time employees number ranging from 30 to 75 staff. In addition, the manufacturing sector definition for the medium and small enterprise have higher sales turnover of RM 15 million to RM 50 million for medium enterprise and RM 300,000 to RM 15 million for small enterprise, respectively.

Due to their size, SMEs are often more adaptable to a new change as compared to the big conglomerates. The data the SMEs accumulate are often more structured and have formats that are easier to 
crunch. With the advancement of technology such as business intelligence (BI), SME can capture insights that can boost its competitive advantage. McKinsey surveyed by interviewing 400 top managers of international companies from a wide variety of industries and the survey result shows that extensive use of customer analytics heavily drives corporate performance with a quantum jump of between 126 to $186 \%$ for profit, sales, sales growth, and return of investment (ROI) [3].

However, SME may face difficulties in data collection and data integration because most companies use multiple tools to gather data, such as; Google analytics, customer relationship management (CRM) tools, social media such as Facebook analytics or flat files such as excel sheets. The data are spread from multiple sources and need to be collectively worked on. The heterogeneity of data has intensely increased, and it is produced in a fast manner making it difficult to manage the data especially if the traditional way of handling data is implemented. Moreover, the lack of efficient visualization in reporting for various stakeholder groups is also making it difficult to assess the current situation. Hence, a solution that can consolidate multiple data sources in one place that is supported by streamlined real-time data is essential [4].

The dashboard is the central interface between a user and business intelligence that acts as a platform to access, interact, analyze, and visualize the analytics data in the form of infographics. It gives a quick overview of multiple metrics simplifying the need to find and analyze data from various data sources. Uniquely, compared to visualization modalities for presentation and exploration, dashboards bring together challenges of at-a-glance reading, coordinated views, tracking data, and both private and shared awareness [5]. The staff's attention can be focused on the content of the data rather than the process of collecting the data resulting in data-driven decisions. The performance can be monitored and measured in real-time using actual data dynamically. The raw data are collected, and relevant insights are extracted and presented in a manageable chunk of visual information for the different groups of the stakeholders that minimize the dependency to the IT assistance staff. The generated reports are dynamic and can be tailored to the need of the stakeholders. This can help managers to make informed decisions that lead to better, more efficient process in the actual work environment and help create a powerful competitive advantage [6].

In this paper, we propose the implementation of the interactive intelligent dashboard (iid-SME) that uses various visualization techniques to highlight the insights that may not be clearly observable before. We focus on the information deliverable in such a way that it can facilitate decision making from a single interface. This paper is organized in the following manner. Section 2 describes the common theoretical concepts used in this work such as business intelligence, visualization techniques, and relevant works by other researchers on SME dashboards for comparative purposes. Then, the methodology of the study is presented in section 3. The implementation of the proposed approach is discussed in Section 4 and this paper is concluded with a summary and future work in section 5.

\section{BUSINESS INTELLIGENCE AND VISUALIZATION}

\subsection{Business intelligence}

The definition of business intelligence (BI) has been outlined on different stakeholders' perspectives, such as; consultancies, software vendors, practitioners, and the scientific community. The term business intelligence has vaguely used to describe processes and systems dedicated to the systematic and purposeful analysis of an organization and its competitive environment [7]. Dresner [7, 8] as cited in [9] defined BI as a broad category of software and solutions for gathering, consolidating, analyzing and providing access to data in a way that allows enterprise users make better business decisions. It is using a data-driven approach utilizing the fact-based decision support system. Forrester defined BI as a set of methodologies, processes, architectures, and technologies that transform raw data into meaningful and useful information used to enable more effective strategic, tactical and operational insights and decision-making that can put a company ahead of its competitors [10]. In a more recent attempt, Muntean [11] considered the BI term to cover the art of picking up business advantage from data by coordinating divergent information sources into a solitary framework for real-time revealing and detailed investigation inside the broadened enterprise. Although Dresner's original definition of BI [7], as well as more recent definitions from analysts like Forrester [10], are broad in scope, most practitioners associate with the term a narrow set of capabilities, such as extraction, transformation, and loading (ETL); data warehousing; on-line analytical processing (OLAP); and reporting [12].

\subsection{Visualization of business intelligence}

Analytics uses the information acquired from BI by modifying it into a meaningful format that leads to relevant insights that can be applied to strategies that help the organization to create and extract value especially in decision-making. In a simpler word, BI deals with what happened in the past and how it happens using trend and pattern identification whereas business analytics (BA) deals with the contributing factors and causality that explains the reason why such a situation happened in the past. BA also uses these 
reasons to predict the future using forecasting and prediction. However, these two concepts are interrelated and interchangeably used to improve business performance. Burley and Ashburn [13] have identified data visualization as the study of the visual representation of data that extract hidden information in some schematic form, including attributes or variables for the units of information. It can facilitate human cognition by manipulating human visual capabilities to make sense of abstract information [14].

Current businesses use data visualization to help users understand the significance of data by placing it in an aesthetical visual context. Insights in terms of patterns, trends, and correlations are easily observable and areas that need attention or improvement are identified as compared to text-based data. The implementation of data visualization allows the user to effectively interpret the data and adjust the different variables to see their effect on the visual representations. It is about the way the data to present to the right people at the right time to enable them to gain insights most effectively. Moreover, visualization enables the user to drill down into important details that allow the user to manipulate the data representation directly with active interaction. Such functionality can be translated into actionable insights for better business performance.

\subsection{Dashboard and visualization tools}

One of the most important visualizations approaches in BI is the dashboard. The dashboard is the main graphical interface used between the user and a BI engine. It presents the most important reports and data visualizations for the user depending on their customized level of access and role. It acts as a platform that allows users to visualize the analytics data in the form of infographic or pictorial format. Wexler et al. defined the dashboard as a visual display of data used to monitor conditions and/or facilitate understanding [15]. Velcu-Laitinen and Yigitbasioglu [16] stated that a dashboard is a diagnostic tool that can present a quick overview of the company's performance. Pauwels et al. outlined the purpose of dashboard usage as the following functions, namely; monitoring, consistency, planning, and communication [17].

When the user first logging to the system, the dashboard is the first view the user sees. Such a concept is adopted from the vehicular dashboard that allows the user to view the overall information in a simplified manner $[18,19]$. Since all the insights can be provided in a single place, the amount of time needed to extract these insights can be reduced and could be utilized to make better analysis resulting in faster and well-informed decisions. The organizations that use visual data discovery are more likely to find timely information than those who rely on traditional methods [20]. Creating ideal measurements, work process, navigation, data source framework, and guaranteeing that data is relevant are the challenges that need to be catered when designing an interactive dashboard.

The dashboard can be customized through the use of visualization tools in facilitating the user's understanding of the data [21]. There are many visualization tools available, ranging from traditional formats, such as; bar graphs, pie charts, and scorecards to more advances data visualization techniques that can discover deeper insights, make predictions, generate a recommendation, create interactive and dynamic content or automatically select the optimal data representation depending on user personalization. There are many good visualization tools, such as Tableau, Qlikview, FusionCharts, and others. Although there are many functionalities and advantages of using either of the data visualization software, no visualization tools can claim superiority compared to the other because each tool has its own uniqueness. In this work, however, we are using Tableau software because of its availability and ease of work.

\section{RESEARCH METHOD}

The work in this paper is segregated into 6 consecutive phases. All the phases are comprise of data collection, data extraction, data loading and pre-processing, data processing, and data analysis. The summary of the overall approach is presented in Figure 1.

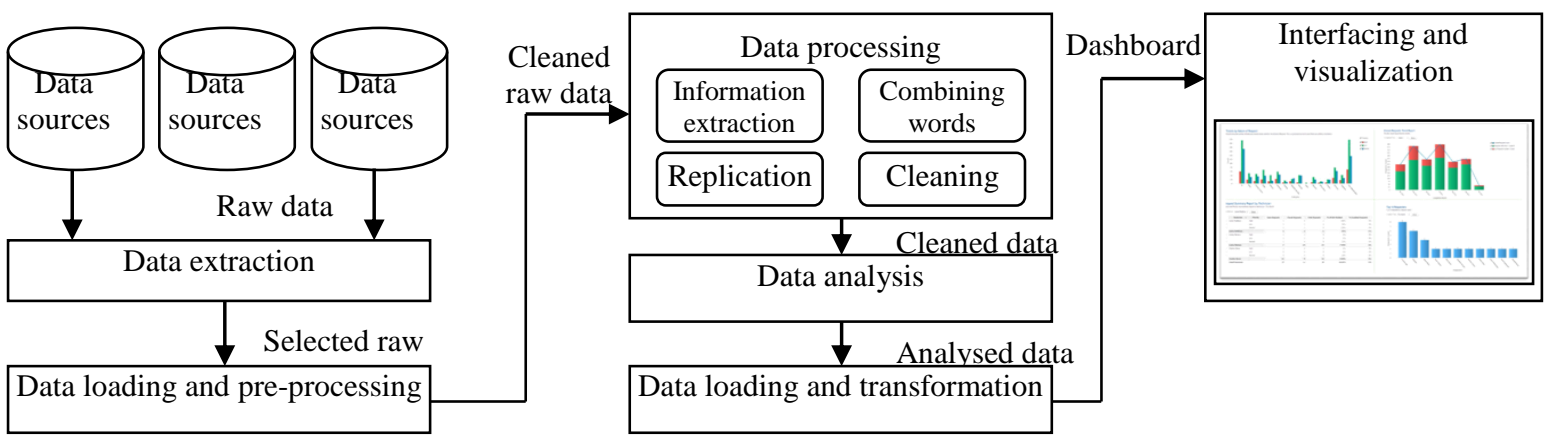

Figure 1. The overall architecture of the proposed solution of iid-SME 
In the first phase, data are collected manually from multiple sources, such as; printed service reports, emails, messages, and chats sessions from social network services that are captured from the SME's cloud. These combinations of structured, semi-structured, and unstructured data need to be manually processed to generate the required data for this study. Typically, the support team contacted clients by email, phone calls, messages, and completion of the printed service report. Such an approach is posed as a challenge to track the data and no action taken since they are not recorded. There is also no evidence and verification of the work completed by the support team. Hence, a mechanism to store the data is essential. For this work, we used log case complaints gathered from email, messages from social network services such as WhatsApp and iMessage, and the information from the printed service report. The data collected in this study is taken from June 2017 until March 2018 manually. Once the data are collected, the summary of the finding and the interpretation of the data are formed.

The raw data and stream data for instance time of the raw data produced segregated from the different sources are extracted in the data extraction phase. Then, the data are transfers and loaded in the data loading and pre-processing phase. Once the data are collected, the data need to be cleaned and pre-processed using operation such as eliminating unnecessary words, combining the replicated words and replicated the necessary words to ensure the meaning of the word will be complete. From there, the analysis of the cleaned data is conducted in the data analysis phase. Once the relevant data are analyzed, the data will be transformed and loaded into the data loading and transformation phase.

The whole overall approach is completed by implementing the interactive dashboard in the visualization and interfacing phase to offer an aesthetically pleasing graphical representation of the processed data to make the insights observable in a fast and easy manner. In this work, an interactive intelligent dashboard for SME is executed. It offers accessibility to business data at one point as well as provides adaptability and straightforwardness in analyzing data. The iid-SME dashboard begins with a complex view level that can summarize the business data. The users can drill down or drill across to the minimal level of the module to get the details of the information. This is especially beneficial to the executive level to get the data perspective from the operational side in real-time. In addition, the iid-SME dashboard can be focused on a specific part based on the user level of access and his/her role. This work is in line with Galesic et al. graph literacy metrics [22] that enable finding and extracting data (L1) and integrating data and finding relation (L2) but still working on analyzing implicit relationships, generating and predicting (L3).

\section{EXPERIMENTAL RESULT AND DISCUSSION}

Tableau allows a variety of data types and users can select the data they want to visualize based on their needs and level of access. In this work, we developed four interfaces with different visualization techniques to give a better understanding of the data.

\subsection{HeatMap}

HeatMap is a graphical representation of data that uses a color-coding system to show the density of the data. In this work, heatMap is used to illustrate the distribution of the customer population in Malaysia. The gradient color represents the total number of customers by state. The concentrated green color denotes the highest number of customers located as opposed to low-density green color that has minimal customers as illustrated in Figure 2. It can be observed that the majority of the customer is populated in central Malaysia that is in Klang Valley or generally can be said in Selangor state.

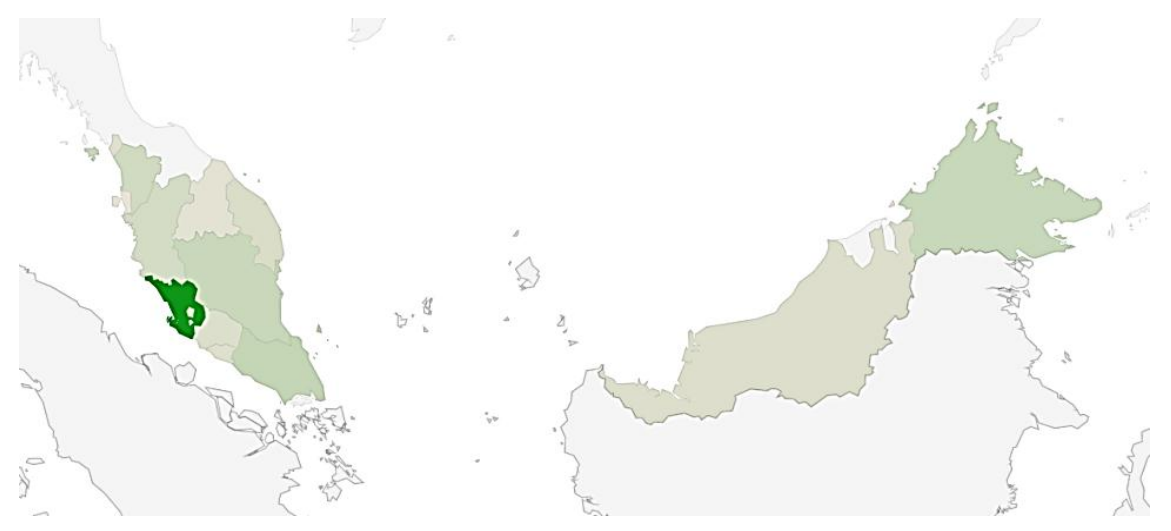

Figure 2. Customers distribution based on geographical location 


\subsection{Line chart}

A line chart helps to compare changes over the same period for more than one group. In this work, we implement the line chart to see the period of ticket expiry given in a month. The data can be filtered using location and distinct count of cases. A ticket is given if the customer reports or complaint about issues that needed IT support. The complaints can be in the form of WhatsApp conversation, printed report or an email. Once the IT support department received the complaint, a ticket will be produced to keep track of the duration needed to handle that task. By having the line chart, users can observe the number of tasks that have been completed by specific staff and the days needed to complete the task. Such information is important to monitor the individual staff performance, the complexity of the task so that the administration can assign more staff to handle complicated issues and make predictions on the customer's problem based on their frequency and consistency of having a similar problem over time. The information can also indicate the need to have in-house IT support or to outsource the service. Figure 3 shows the line chart for ticket duration by month.

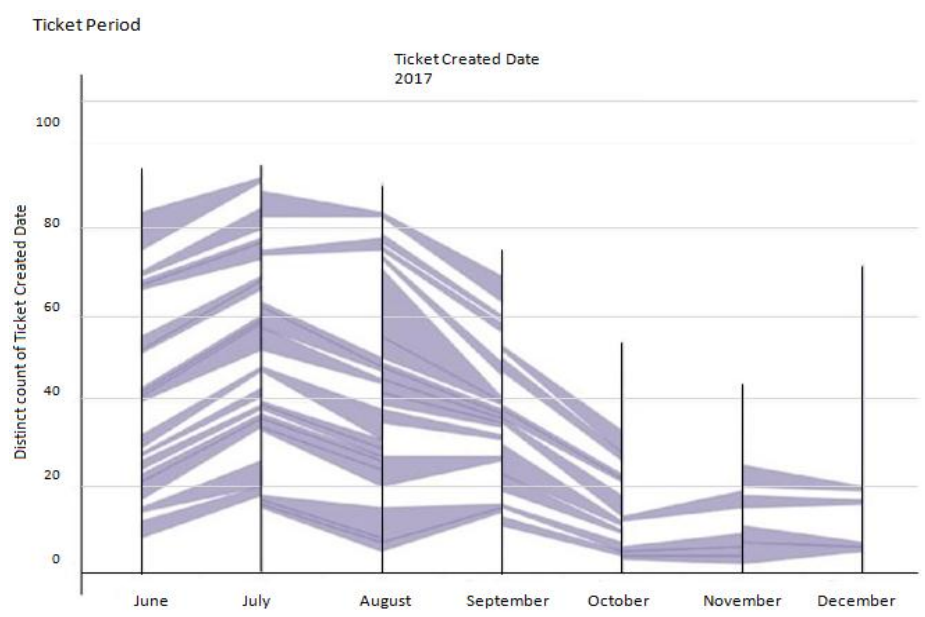

Figure 3. Ticket period in a month

\subsection{TreeMap}

TreeMap comprises of a series of rectangles to show hierarchical data. Figure 4 depicts the channel used by the customer to communicate with the company. Each block represents the type of user-preferred channel for communication, such as; on-site, WhatsApp, phone call, online chat, TeamViewer, email, and web communication. The size of the block specifies the number of channel usage. The bigger the size of the box, the higher the number of customers using the channel to communicate. It is good for comparing proportions within the hierarchy to the whole. From Figure 4 it is clear that the customers prefer to use phone calls and chat when reporting the issue to the IT support (represented in bright and dull pink colors) and minimal engagement is made using the website (denoted in dark grey color). Hence, an improvement to the company website is needed to allow better communication with the customers.

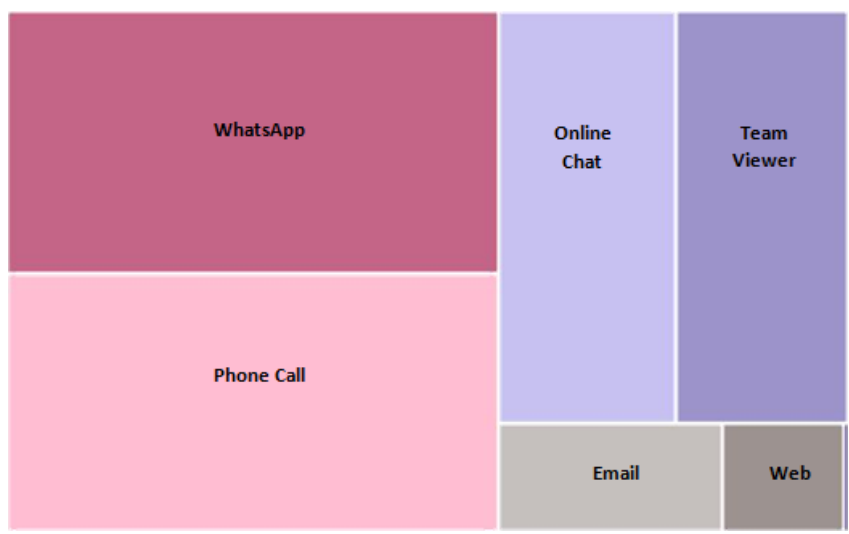

Figure 4. User preferred channels for communication 


\subsection{Sunburst chart}

Sunburst chart visualization shows hierarchy through a series of rings that correspond to a level in the hierarchy. The innermost ring represents the top hierarchy while the outermost ring represents the last hierarchy. Figure 5 shows the relationship between the tickets owners versus the number of cases handled. Different colors representing different engineers who handled the tasks. The thickness of the circle represents the individual number of the cases handled. The diagram shows the performance of the engineer and his/her appraisal assessment can be based on this. Subsequently, many opportunities can be created to further enhance the company's need to measure the performance of the staff using the proposed iid-SME approach in various stages [23-26]. The proposed visualization techniques further enhanced the management understanding of critical factors governing the success of the company.

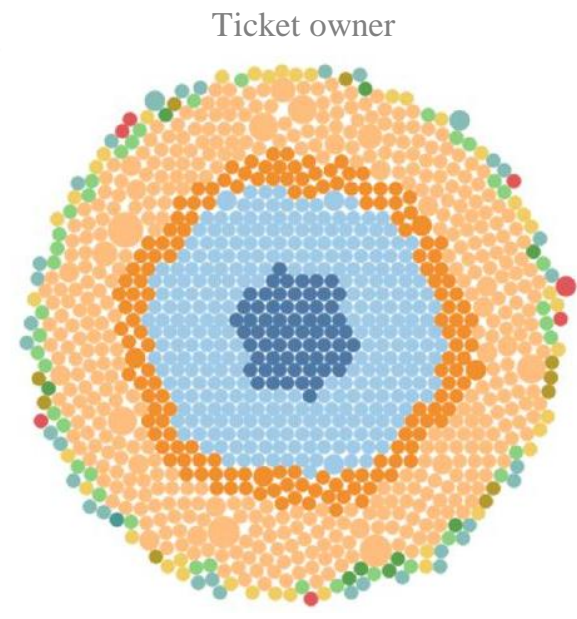

Figure 5. Cases handled by engineers

\section{CONCLUSION}

Business Intelligence (BI) can provide advanced and predictive information as to the company's performance. The solution shows the potential of highlighting hidden insights that can be used to make better-informed decisions. In fact, the use of blockchain technology for the purpose of profiling in building portfolios can be used to ensure verification and validation of the portfolio is secured for the company or each individual employee. Thus, applications of BI are vast and huge limited only by the minds.

\section{ACKNOWLEDGMENTS}

The authors would like to thank the Faculty of Computer and Mathematical Sciences (FSKM), Universiti Teknologi MARA (UiTM) and Kulliyah of Information and Communication Technology, International Islamic University Malaysia (IIUM) for providing financial support to conduct the work published in this paper.

\section{REFERENCES}

[1] M. Madanchian, N. Hussein, F. Noordin, and H. Taherdoost, "The role of SMEs in economic development: Case study of Malaysia," International Journal of Academic Research in Management, vol. 4, no. 3, pp. 77-84, 2015.

[2] SME Corp. Malaysia, "Small and medium enterprises," Mar. 2020. [Online]. Available at: http://www.smecorp.gov.my/index.php/en/small-and-medium-sized-enterprises.

[3] A. Bokman, L. Fiedler, J. Perrey, and A. Pickersgill, "Five facts: How customer analytics boosts corporate performance," McKinsey \& Company, 2014 [Online]. Available at: https://www.mckinsey.com/businessfunctions/marketing-and-sales/our-insights/five-facts-how-customer-analytics-boosts-corporate-performance.

[4] J. Jordan and C. Ellen, "Business need, data and business intelligence," Journal of Digital Asset Management, vol. 5, no 1, pp. 10-20, 2009.

[5] A. Sarikaya, M. Correll, L. Bartram, M. Tory, and D. Fisher, "What do we talk about when we talk about dashboards?," IEEE Transactions on Visualization and Computer Graphics, vol. 25, no 1, pp. 682-692, 2018

[6] E. Foley and M. G. Guillemette, "What is business intelligence?," International Journal of Business Intelligence Research (IJBIR), vol. 1, no 4, pp. 1-28, 2010.

[7] H. Dresner, "Business intelligence," Gartner Inc., 1989

[8] H. Dresner, "Profiles in Performance: Business intelligence journeys and the roadmap for change," John Wiley \& Sons, 2009. 
[9] L. Buchanan and A. O Connell, "A brief history of decision making," Harvard Bus. Rev., vol. 84, no 1, p. $32,2006$.

[10] C. Elena, "Business intelligence," J.of Knowl. Manag., Eco. and Inf. Tech., vol. 1, no. 2, pp. 1-12, 2011.

[11] M. Muntean, "Theory and practice in business intelligence," SSRN Electronic Journal, vol. 2144440, pp. 1-6, 2012.

[12] T. H. Davenport, "Competing on analytics," Harvard Business Review, vol. 84, no.1, pp. 98-107, 2006.

[13] D. Burley and V. Ashburn, "Information visualization as a knowledge integration tool," Journal of Knowledge Management Practice, vol. 11, no. 4, p. 1, 2010.

[14] M. Card, "Readings in information visualization: Using vision to think," Morgan Kaufmann Publishers, 1999.

[15] S. Wexler, J. Shaffer, and A. Cotgrave, "The big book of dashboards: Visualizing your data using real-world business scenarios," John Wiley \& Sons, 2017

[16] O. Velcu-Laitinen and O. M. Yigitbasioglu, "The use of dashboards in performance management: Evidence from sales managers," International Journal of Digital Accounting Research, vol. 12, pp. 39-58, 2012.

[17] K. Pauwels, T. Ambler, B.H. Clark, P. LaPointe, D. Reibstein, B. Skiera, B.Wierenga, and T. Wiesel, "Dashboards as a service: Why, what, how, and what research is needed?," J. of Serv. Research, vol. 12, no 2, pp. 175-189, 2009.

[18] A. Marcus, "Dashboards in your future," Interactions, vol. 13, no 1, pp. 48-60, 2006.

[19] O. M. Yigitbasioglu and O. Velcu, "A review of dashboards in performance management: Implications for design and research," International Journal of Accounting Information Systems, vol. 13, no 1, pp. 41-59, 2012.

[20] D. Keim, G. Andrienko, J. D. Fekete, C. Görg, J. Kohlhammer, and G. Melançon, "Visual analytics: Definition, process, and challenges," Information Visualization. Springer, Berlin, Heidelberg, pp. 154-175, 2018.

[21] N. N. Ramly, F. M. Nor, N. H. Ahmad, and M. H. Aziz, "Comparative analysis on data visualization for operations dashboard," International Journal of Information and Education Technology, vol. 2, no 4, pp. 287-290, 2012.

[22] M. Galesic and R. Garcia-Retamero, "Graph literacy: A cross-cultural comparison," Medical Decision Making, vol. 31, no. 3, pp. 444-457, 2011

[23] N. Kamaruddin, A. W. A. Rahman, and A. N. R. Shah, "Measuring customer satisfaction through speech using valence-arousal approach," The 6th Int. Conf. on Inf. and Comm. Tech. for The Muslim World, pp. 298-303, 2016.

[24] N. A. M. Zamani, N. Kamarudin, A. Wahab, and N. S. Saat, "Visualization of job availability based on text analytics localization approach," Indonesian Journal of Electrical Engineering and Computer Science, vol. 16, no. 2, pp. 744-751, 2019.

[25] M. Bakri, N. Kamaruddin, M. Hamiz, P. Marlia, A. H. S. Nurhasmiza, A. B. Shahirah, Z. Othman, N. A. S. Nilam, M. S. O. Shahir, S. A. H. Nurul, L-C. L. Effie, B. Nigel, and H. Matthias, "Insights extraction on cross-cultural interaction through astronomy online labs using data analytics", Indonesian Journal of Electrical Engineering and Computer Science, vol 16, no 1, pp. 508-515, 2019

[26] N. Kamaruddin, A. W. A. Rahman, and R. A. M. Lawi, "Jobseeker-industry matching system using automated keyword selection and visualization approach", Indonesian Journal of Electrical Engineering and Computer Science, vol. 13, no. 3, pp. 1124-1129, 2019.

\section{BIOGRAPHIES OF AUTHORS}

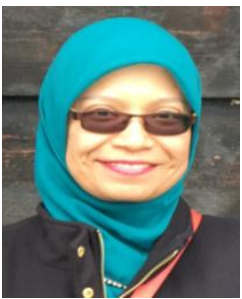

Norhaslinda Kamaruddin currently holds a post of associate professor in Faculty of Computer and Mathematical Sciences, Universiti Teknologi MARA (UiTM), Malaysia. She served UiTM since 2011. She received her bachelor's degree in Information Technology (Computer Science) from Universiti Kebangsaan Malaysia in 2001 followed by her Master of Software Engineering from Malaya University in 2006. In 2013, she is awarded a Doctor of Philosophy (Computer Engineering) from Nanyang Technological University (Singapore) focusing on computational intelligence especially on Affective Computing. She is very active in the research fields of affective computing, speech emotion recognition, neuro-cognitive informatics, big data analytics, and driver behavioral study.

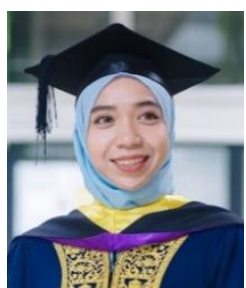

Raja Durratun Safiya received her bachelor's degree in Data Communication and Networking (Computer Science) from University Teknologi MARA (UiTM), Malacca, Malaysia and completed her master's degree in Web Technology (Computer Science) from University Teknologi MARA (UiTM) Shah Alam, Malaysia. Her attentiveness in network analysis and network security system. She has been involved in Cisco Certified Network Associate (CCNA) and presently active in the professional field in Microsoft Certified Systems Engineer (MCSE).

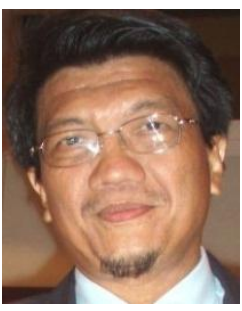

Abdul Wahab received a Degree from the University of Essex, Essex, U.K., in 1979, the M.Sc. degree from the National University of Singapore, Singapore, in 1987, and the Ph.D. degree from Nanyang Technological University, Singapore. His research has been in the areas of telecommunication, signal processing, and artificial intelligence. He was with Hewlett Packard Singapore, Singapore, as a Research and Development Project Manager both in CO, USA. He joined Nanyang Technological University in 1990, where he was an Associate Professor, before joining the International Islamic University of Malaysia, Malaysia, as a Professor, in 2009. $\mathrm{He}$ has authored over 100 conference papers, journal papers, patents, and book chapters in the areas of digital and optical computing, signal processing, and artificial intelligence. 